\title{
Planning and preparation for pregnancy among women with and without a history of infertility
}

\author{
Natalia Ignaszak-Kaus, Katarzyna Ożegowska, Piotr Piekarski, \\ Leszek Pawelczyk, Piotr Jędrzejczak
}

Department of Infertility and Reproductive Endocrinology, Poznan University of Medical Science, Poznan, Poland

\begin{abstract}
Objectives: Preconception counseling, maternal health-related habits, diet, folic acid consumption, substances abuse, may all impact the outcome of pregnancy. The aim of this study was to compare the planning and preparation for pregnancy among pregnant women with and without infertility.

Material and methods: A survey of health behaviors prior to and during pregnancy that could affect pregnancy outcomes, including laboratory tests performed, stimulant usage, initiation of prenatal care, and folic acid intake, was conducted among 400 pregnant women. The study group included 121 women $(30.25 \%)$ diagnosed with prior infertility, while the control group included 279 women (69.74\%) who did not report any problems conceiving.

Results: All patients (100\%) from the study group and 70,97\% from the control group planned their pregnancy $(p<0.0001)$. Patients in the study group performed significantly more laboratory tests prior to pregnancy, including: complete blood count, urine analysis, fasting blood glucose concentration, testing for toxoplasmosis, and Pap smear, compared with the control group $(p<0.0001)$. There was no difference between groups regarding the knowledge of when and why folic acid supplementation is required ( $p>0.05$ ).

Conclusions: Effective education of women, regarding pregnancy planning and behaviours, that may impact pregnancy outcome is still a serious challange to public health in Poland. Our study indicates that reaching general population with the education is most important to achieve best results in preconceptional care.
\end{abstract}

Key words: infertility, pregnancy planning, folic acid, prevention

Ginekologia Polska 2018; 89, 2: 74-79

\section{INTRODUCTION}

It is well known that the first weeks of pregnancy are of great importance for fetal development and pregnancy outcomes. Unfortunately, a majority of women are not aware of being pregnant during this period, and, thus, may not provide an adequate healthy lifestyle. Therefore, pregnancy planning, preconception counseling, and ensuring the mother's health prior to pregnancy are important for positive pregnancy outcomes [1]. Studies have been published that prove the effects of folic acid, drugs, and general health of the woman on pregnancy and the developing fetus [2]. However, it is not clear whether all women are aware of this information and are properly preparing for their pregnancy. Many studies emphasize that the rate of unplanned pregnancy is still high, even in developed countries [3].
A study from the Centers for Disease Control and Prevention (2012) revealed that $49 \%$ of women did not receive proper preconception counseling and that their pregnancy outcomes were worse compared with those who had received complete preconception counseling. Therefore, this study highlighted the necessity of preconception care for proper pregnancy outcomes [4]. Preconceptional care combines 3 major aims: evaluation of risk factors, health promotion, and health intervention [5] .

The World Health Organization (WHO) defines infertility — the inability to become pregnant and have a baby despite regular sexual intercourse without contraception for at least 12 months - as a disease [6]. Although there is a need for pregnancy planning and preconception care in the general population, women with infertility should be, and usually 
are, evaluated much more precisely in order to diagnose the reason for the lack of pregnancy. The prevalence of primary infertility have been reported $0.6 \%$ to $3.4 \%$, and secondary infertility is $8.7 \%$ to $32.6 \%[7,8]$.

There are several ways to treat infertility. Unfortunately, most methods are not cost-effective, have a high failure rate, and often carry some risks for the patient. Moreover, most infertile couples have a higher preliminary risk than healthy individuals based on age, obesity, hormonal treatment, and/or chronic disease [5]. Because all these factors may lead to unsuccesful infertility treatment and poor pregnancy outcomes, each health care activities should lead to improvement of infertility treatment and help to increase the chance of positive pregnancy outcomes. Preconception counseling in infertile couples can increase the chance of pregnancy and lower its complications [9-11]. Because pregnancy failure may lead to various psychological, financial, and mental complications, relatively cheap and easy preconception interventions are recognized as a convenient, cost-effective, and practical factor for reduction of risk factors and increase in pregnancy success. Therefore, this study was designed to evaluate the trend of preconception care in women diagnosed with infertility who tried to get pregnant for at least one year either without medical advice or by undergoing any type of medical treatment.

The aim of this study was to compare the planning and preparation for pregnancy among pregnant women with and without a history of infertility. We also assessed the knowledge about the usefulness of basic laboratory tests, the importance of folic acid supplementation, and the harmfulness of certain drugs and stimulants among these groups of patients. We wanted to investigate health-relataed behaviours of pregnant women with and without the history of infertility.

\section{MATERIAL AND METHODS}

We analyzed data from a survey conducted among pregnant women who were hospitalized at the Poznan Obstetrics and Gynecology Teaching Hospital. The survey of health behaviors prior to and during pregnancy that could affect pregnancy outcomes, including laboratory tests performed (complete blood count, urinalysis, fasting blood glucose, toxoplasmosis [immunoglobulin (lg) G and IgM], Rubella [lgG], and Pap smear), alcohol consumption, cigarette smoking, initiation of prenatal care, and folic acid intake, was conducted by trained interviewers to ensure proper understanding of the questions. The study group included pregnant women diagnosed with infertility who underwent any infertility treatment (e.g., surgery, ovarian stimulation, assisted reproductive techniques) in order to get pregnant. The control group included pregnant women who did not report any problems conceiving. The groups were age-matched. The study was approved by the Institutional Review Board of Poznan University of Medical Sciences, and all patients provided written informed consent.

Statistical analysis was performed by using Statistica 10.0 (StatSoft, Inc., Tulsa, OK, USA). The normality of the distribution of variables was tested by using the Kolmogorov-Smirnov test. For comparisons between groups, the chi-square test and Mann-Whitney $U$ test were performed. $P=0.05$ was accepted as the cut-off value for statistical significance.

\section{RESULTS}

Four hundred patients were included in the study. The study group included 121 women (30.25\%), while the control group included 279 (69.74\%). Both groups did not differ significantly according to age, place of residence, or level of education ( $p>0.05)$. The average age in both groups was 30 years (range, 16-44 years). Statistically, average age of pregnant patients with history of infertility is usually higher than in health population. The groups were age-matched. We did not want to compare patients of different age, because the knowledge about reproductive health among older women is generally greater.

\section{Planning and preparation for pregnancy}

In the study and control groups, $100 \%$ and $70.97 \%$ planned their pregnancy, respectively $(p<0.0001)$. Pregnancy planning including preconception examination and counseling was performed in 119 women (98.35\%) in the study group, whereas, in the control group, only 144 women $(51.61 \%)$ regularly met with a gynecologist $(p<0.0001)$. Patients in the study group performed significantly more laboratory tests prior to pregnancy, including complete blood count, urine analysis, fasting blood glucose, toxoplasmosis, and Pap smear, compared with the control group $(p<0.0001)$. There was no significant difference in rubella testing between both groups ( $p=0.07$ ) (Tab. 1).

\section{Folic acid supplementation}

In the present study, we also evaluated the awareness of folic acid supplementation. There was no significant difference between groups regarding the knowledge of when and why folic acid supplementation is required ( $p>0.05$ ). One hundred eight women (89.26\%) in the study group and $238(85.30 \%)$ in the control group knew that usage of folic acid corellates with lower rates of fetal malformations. The recommendation of at least 6 weeks of preconceptional folic acid supplementation was known by 100 women (82.64\%) in the study group and by 190 (68.10\%) in the control group $(p>0.05)$. Although the knowledge of the need for folic acid supplementation was not different between the groups, there was a significant difference in the rate of folic acid 
Table 1. Percentage of basic laboratory tests performed in each group

\begin{tabular}{|l|c|c|}
\hline Recommended examination & Study group & Control group \\
\hline Complete blood count* & $91(75.21)$ & $95(34.05)$ \\
\hline Urinalysis* & $76(62.81)$ & $75(26.88)$ \\
\hline Pap smear* & $91(75.21)$ & $114(40.86)$ \\
\hline Fasting blood glucose* & $35(28.93)$ & $40(14.34)$ \\
\hline Toxoplasmosis (lgG/lgM)* & $45(37.19)$ & $40(14.34)$ \\
\hline Rubella $(\mathrm{lgG})$ & $10(8.24)$ & $14(6.76)$ \\
\hline
\end{tabular}

Data are expressed as $n(\%)$ ${ }^{*} p<0.0001$

\begin{tabular}{|l|c|c|}
\begin{tabular}{l} 
Table 2. Frequency of folic acid intake in each group \\
\hline Folic acid intake*
\end{tabular} & Study group & Control group \\
\hline None & $19(15.70)$ & $98(35.13)$ \\
\hline Occasional & $9(7.44)$ & $13(4.66)$ \\
\hline $1-3$ times/week & $3(2.48)$ & $15(5.38)$ \\
\hline $4-6$ times/week & $5(4.13)$ & $20(7.17)$ \\
\hline Daily & $85(70.25)$ & $133(47.66)$ \\
\hline
\end{tabular}

Data are expressed as $\mathrm{n}(\%)$

${ }^{*} p<0.0001$

\begin{tabular}{|l|l|c|c|}
\hline \multicolumn{2}{|c|}{ Table 3. Use of stimulants in each group } \\
\hline \multirow{3}{*}{ Smoking } & Hazard awareness* & Study group & Control group \\
\hline & Before pregnancy & $110(90.90)$ & $231(82.80)$ \\
\cline { 2 - 4 } & During pregnancy & $22(18.18)$ & $66(23.66)$ \\
\hline \multirow{3}{*}{ Alcohol } & Discontinue prior to pregnancy & $2(1.65)$ & $16(5.73)$ \\
\hline & Discontinue at moment of pregnancy diagnosis & $91(75.21)$ & $201(72.04)$ \\
\hline & During pregnancy & $30(24.79)$ & $78(27.96)$ \\
\hline
\end{tabular}

Data are expressed as $\mathrm{n}(\%)$

${ }^{*} p<0.0001$

intake (Table 2). Compared with the control group, a higher percentage of women in the study group took folic acid as recommended $(0.4 \mathrm{ng}$ per day at least until the second trimester of pregnancy ["Recommendations for," 1992], $\mathrm{p}<0.0001)$. Daily folic acid intake was reported in 85 women (70.25\%) in the study group and in $133(47.66 \%)$ in the control group $(p<0.0001)$. Nineteen patients $(15.7 \%)$ in the study group and 98 (35.13\%) in the control group did not supplement their diet with folic acid during pregnancy (Tab. 2).

\section{Stimulants: alcohol and nicotine}

Awareness of the harmfulness of nicotine usage was slightly higher in the study group than in the control group (Tab. 3). The high percentage of the studied group responded that one should stop smoking at the time of making the decision to become pregnant (110 [90.90\%] in the study group vs 231 [82.80\%] in the control group, $p=0.045$ ). However, the percentage of women who smoked before and during pregnancy were comparable between groups ( $p>0.05$ ). In addition, a similar percentage of women in both groups expressed the opinion that alcohol should be discontinued at the time of pregnancy planning, and that it should be abandoned until the pregnancy is finished ( $p>0.05)$. Two patients (1.65\%) in the study group and $5(1.79 \%)$ in the control group admitted that they happened to drink alcohol during pregnancy $(p>0.05)$ (Tab. 3).

\section{DISCUSSION}

Preconceptional planning, every-day behaviours of women, may increase the chance of successful pregnancy and decreases its complications. All women should plan their pregnancy, but infertile couples may particularly 
benefit from preconceptional care and counseling. Although it is known to be beneficial when the pregnancy is planned, the rate of unplanned pregnancies is still high. In this study, 81 pregnant women (29.03\%) without infertility confirmed that their pregnancy was not planned at all. Similar results were presented in a study evaluating the decisions about becoming pregnant among women in a clinic in Berlin [3]. In that study, 33\% of women didn't plan their pregnancies. Similar results also were obtained from a large multicenter study in London, in which only $73 \%$ of pregnant women reported that their pregnancy was the result of conscious decision [12]. Still in too many cases, the decision about pregnancy is not followed by the proper preparation. In our study, the majority of patients (70.97\%) with a history of infertility were under the care of a gynecologist before pregnancy, whereas, in the group of women who conceived spontaneously, that number was only slightly more than half (51.61\%). Therefore, there was a large discrepancy between the planning and preparation for pregnancy among women without infertility. Our results coincide with those obtained in a large study presented by Stephenson et al. [12], in which the rates of pregnancy planning and medical counseling were $73 \%$ and $51 \%$, respectively.

Our study presented that more basic laboratory tests were performed in the group with infertility, that might have been expected, because that group of women is more aware of its' potential risks. In both groups, the most common test that was done is Pap smear, that was performed in $75.21 \%$ of women with infertility and in $40.86 \%$ of women without. These results are not satisfactory, because cytology testing should be mandatory $[13,14]$. However there are studies presenting data that $76.8 \%$ of pregnant women did not undergo a Pap smear regularly before pregnancy [15]. These findings show that cervical cancer prevention, although easy and effective, is still insufficient in many countries.

The second most commonly performed test was a complete blood count, which was performed in $75.21 \%$ of women with infertility and in only $34.05 \%$ of women without infertility. These results are unacceptable considering the fact that maternal anemia may be associated with significantly increased risk of low birth weight and low Apgar score [16]. Moreover, low hemoglobin concentration in the first trimester of gestation seems to have the most detrimental effect on fetal outcomes [17]. It has been demonstrated that treatment of anemia before and in the first trimester of pregnancy can prevent adverse pregnancy outcomes. So detection of anemia in the early stage of pregnancy is possible only in women who plan and prepare for their pregnancy. In our study, women with a history of infertility treatment had a better opportunity for faster diagnosis and treatment.

Urine analysis was performed in $62.81 \%$ of women with infertility and in only $26.88 \%$ of women without infertility.
High percentage of abnormal results among studied patients confirm the need to perform a urine culture test to detect asymptomatic bacteriuria, which is a known risk factor for both preterm delivery and low birth weight [16]. It strongly proves that urine testing should be performed routinely in all pregnant women regardless of the presence of symptoms.

Evaluation of fasting blood glucose was performed in $28.93 \%$ of women with infertility and in $14.34 \%$ of women without infertility. Congenital defects typical for diabetes, such as caudal regression syndrome or central nervous system, often are due to hyperglycemia before pregnancy especially among obese women and not detected type 2 diabetes [18]. Many studies demonstrate the importance of pregnancy planning in women with pregestational diabetes, but also among women with other metabolic disturbances. It turned out that both normalization of glucose levels during pregnancy and neonatal outcomes were better in women who planned their pregnancy $[18,19]$. Maintaining normal glycemia, especially in the first trimester, is essential for preventing congenital defects [18]. Highlighting the significance of planning and proper preparation for pregnancy in women belonging to the risk groups, in our study, the higher prevalence of pregnancy planning and fasting blood glucose testing in women with infertility suggests potentially better pregnancy outcomes in this group of patients.

In our study, toxoplasmosis antibodies were tested in $37.19 \%$ of women with infertility and in $14.34 \%$ of women without infertility. A study by Ripabelli et al. [20], conducted among 2226 pregnant women, showed that only 1151 women (51.7\%) were seropositive. Among these patients, 29 (1.3\%) presented an IgM reagent with high-avidity IgG. In another study conducted in Thailand, anti-Toxoplasma IgG and IgM antibodies were found in $25.0 \%$ and $3.0 \%$ of pregnant women, respectively [21]. Due to the low percentage of women with positive lgG antibodies against toxoplasmosis and the comparatively high percentage with IgM antibodies, this test is strongly recommended in women who are planning to become pregnant [13].

Interestingly, there was no difference in terms of rubella antibody testing between studied groups. In both groups, the percentage of performed tests was very low: $8.24 \%$ in women with infertility and $6.76 \%$ in women without infertility. A study by Miller et al. [4] proved that rubella infection in the mother before the 11 th week of gestation was associated with the highest rate of heart defects as well as congenital deafness of the fetus. These results are an argument of evaluating the level of immune antibodies or at least checking if the patient was vaccinated against it. In another study, Wang et al. [16] assessed the prevalence of measles-mumps-rubella seropositivity according to age in 1015 participants. Rubella 
seropositivity was only $82.5 \%$ in the 20 -29-year-old group. In addition, in the same age group, rubella seropositivity was significantly lower in women than in men, that strongly proves the need of rubella testing [22].

Neural tube defects arise between 21 and 28 days after fertilization [17] — the period of pregnancy when women often are not aware that they are pregnant. Therefore, folic acid should be added to the diet of all women of reproductive age. Our results show that the knowledge of the function of folic acid and its recommended period of supplementation was appropriate for both groups, but the realization of this recommendation was poorer in women without infertility. This finding certainly has a strong association with pregnancy planning in our group of patients. In a large multicenter study conducted in 18 European countries, $60 \%$ of women claimed that they got pregnant without first consulting their doctor prior to pregnancies [23]. In addition, only $40 \%$ of women knew that folic acid is beneficial for the fetus, and only $17 \%$ knew that the usage of it reduces the risk of neural tube defects [23]. Other studies revealed that infertile couples are more aware of the need of folic acid taking, but such awareness is not necessarily connected with its regular consumption [24].

The negative impact of alcohol on the fetus has been described repeatedly; its most severe form is Fetal Alcohol Syndrome [25]. Cigarette smoking also is contraindicated in pregnancy and has been proved to be a risk factor for miscarriage, premature birth, and low birth weight [2]. Numerous studies also have shown that if smoking cessation is not done before pregnancy, success of infertility treatment is decreased [18].

In our study, the knowledge of the risk of alcohol intake was comparable between groups, while the dangers of nicotine were slightly better known in women with infertility $(p=0.045)$. Unfortunately, only a quarter of the women in both groups believed that alcohol should be discontinued at the moment of confirmation of pregnancy. Slightly better results were obtained in terms of awareness of nicotine usage. It is comforting that only a few patients in both groups had reached for a cigarette during pregnancy. Although the percentage of women who drank during pregnancy was very low $(<2 \%)$, we do not know how many of those, who did not plan their pregnancy, drank during the time of conception and in the early weeks of pregnancy. Therefore, the role of pregnancy planning should be emphasized once again in terms of eliminating risky behaviors, such as drinking alcohol and smoking cigarettes. This is particularly important in women without infertility because the percentage of unplanned pregnancies was significantly higher in this group.

In a population-based study conducted in Brazil among 7572 women, the prevalence of smoking before pregnancy decreased from 28\% in 2007 to $22 \%$ in 2013 ( $p<0.001$ ). The prevalence of smoking during pregnancy also decreased from 22\% in 2007 to $18 \%$ in 2013 ( $p<0.001$ ) [19]. In a cross-sectional survey conducted in Spain among 1004 pregnant women between 12 and 18 weeks of gestation, $60 \%$ of women smoked and $72 \%$ drank alcohol before pregnancy. They also found that $48 \%$ of smokers stopped smoking and $37 \%$ of drinkers stopped drinking during pregnancy [19]. These results are still unsatisfactory. In a web-based study evaluating 8344 pregnant women and new mothers in 15 European countries from October 2011 to February 2012, 35.3\% of women used to smoke before pregnancy and $26.2 \%$ continued smoking during pregnancy. Women who were most likely to smoke before and during pregnancy tended to have a lower education level and lower ratio of unplanned pregnancies. The same group of women also did not take folic acid as recommended and consumed alcohol during pregnancy [26]. In another study conducted in Taiwan among 806 women who had just given birth, $26.6 \%$ of women drank alcohol at any stage after recognition of pregnancy [27]. In a study done by Stephenson et al., in the 3 months before pregnancy, 21\% of women smoked and $61 \%$ drank alcohol. During pregnancy, only $48 \%$ quit smoking and $41 \%$ gave up cigarettes [12].

Comparing those studies, our results indicate a much lower percentage of pregnant women who smoke and drink alcohol.

Our study compare the knowledge between two groups: health and women with history of infertility treatment. It would be interesting to assess knowledge between three groups: health patients who are preparing for pregnancy, health patients who not preparing and women with history of infertility. We would like to raise this issue in the future.

\section{Applications}

This study was carried out to investigate the preconception care and prenatal counseling among women with infertility. Chronic diseases, infections, and abnormal laboratory test results may increase the risk of infertility and pregnancy-related complications. Because infertility may be caused by several medical conditions, prenatal counseling may be much more important in this group of patients. The rates of pregnancy and the mother's mortality due to chronic disease are improvable through better preconception care (Yakir, Riskin-Mashiah, Lavie, \& Auslender, 2011). In infertile couples, preconception care should be carried out with specific attention due to the importance of pregnancy and its good outcome. Specific conditions that may affect infertility treatment should be assessed before pregnancy in order to introduce specific interventions.

In this study, there was a significant difference regarding the planning and preparation for pregnancy between 
patients with and without infertility. Although the knowledge of reproductive health was better among patients with infertility, the level is still unsatisfactory.

\section{CONCLUSIONS}

- Our study showed low knowledge of the need for pregnancy planning and performing of laboratory tests to prevent complications.

- It was lower in a group of healthy women without infertility problems.

- Patients with infertility were more aware of the need for pregnancy planning, and performed more laboratory tests to prepare for pregnancy, but still their knowledge about the role of this tests is unsufficient.

\section{Implications for practice and/or policy}

This is the first study in Poland and one of the few in the world to compare the planning and preparation for pregnancy in women with infertility. There is a need for further studies in this group of patients. Studies showing a correlation between preparation for pregnancy and obstetrical outcomes also would be valuable. It is necessary to improve public awareness of the significance of proper planning and preparation for pregnancy.

\section{Conflict of interest}

None declared.

\section{Acknowledgements}

This work was supported by the grant from the Polish National Center of Science [grant number UMO-2012/07/N/NZ5/00069].

\section{REFERENCES}

1. Wade GH, Herrman J, McBeth-Snyder L. A preconception care program for women in a college setting. MCN Am J Matern Child Nurs. 2012; 37(3): 164-70; quiz 170, doi: 10.1097/NMC.0b013e31824b59c7, indexed in Pubmed: 22417917.

2. Jaddoe VWV, Troe EJWM, Hofman A, et al. Active and passive maternal smoking during pregnancy and the risks of low birthweight and preterm birth: the Generation R Study. Paediatr Perinat Epidemiol. 2008; 22(2): 162-171, doi: 10.1111/j.1365-3016.2007.00916.x, indexed in Pubmed: 18298691.

3. Mikolajczyk R, Rauchfuss M, Lamm D. Unplanned pregnancies in a developed society--a study on a maternity ward in Berlin. Zentralbl Gynakol. 2001; 123(10): 578-584, doi: 10.1055/s-2001-19087, indexed in Pubmed: 11753812

4. Recommendations for the use of folic acid to reduce the number of cases of spina bifida and other neural tube defects. MMWR Recomm Rep. 1992; 41(RR-14): 1-7, indexed in Pubmed: 1522835.

5. Yakir S, Riskin-Mashiah S, Lavie O, et al. Medical evaluation prior to fertility treatment--time for reconsideration? Harefuah. 2011; 150(11): 844-8, 875, indexed in Pubmed: 22428205.

6. Balasch J. Investigation of the infertile couple: investigation of the infertile couple in the era of assisted reproductive technology: a time for reappraisal. Hum Reprod. 2000; 15(11): 2251-2257, indexed in Pubmed: 11056115.

7. Bushnik T, Cook JL, Yuzpe AA, et al. Estimating the prevalence of infertility in Canada. Hum Reprod. 2012; 27(3): 738-746, doi: 10.1093/humrep/der465, indexed in Pubmed: 22258658.
8. Mascarenhas $\mathrm{MN}$, Cheung $\mathrm{H}$, Mathers $\mathrm{CD}$, et al. Measuring infertility in populations: constructing a standard definition for use with demographic and reproductive health surveys. Popul Health Metr. 2012; 10(1): 17, doi: 10.1186/1478-7954-10-17, indexed in Pubmed: 22938182.

9. Bombard JM, Robbins CL, Dietz PM, et al. Preconception care: the perfect opportunity for health care providers to advise lifestyle changes for hypertensive women. Am J Health Promot. 2013; 27(3 Suppl): S43-S49, doi: 10.4278/ajhp.120109-QUAN-6, indexed in Pubmed: 23286663.

10. Twigt JM, Bolhuis MEC, Steegers EAP, et al. The preconception diet is associated with the chance of ongoing pregnancy in women undergoing IVF/ICSI treatment. Hum Reprod. 2012; 27(8): 2526-2531, doi: 10.1093/humrep/des157, indexed in Pubmed: 22593431.

11. van der Zee B, de Beaufort I, Temel S, et al. Preconception care: an essential preventive strategy to improve children's and women's health. J Public Health Policy. 2011; 32(3): 367-379, doi: 10.1057/jphp.2011.13, indexed in Pubmed: 21808249.

12. Stephenson J, Patel D, Barrett G, et al. How do women prepare for pregnancy? Preconception experiences of women attending antenatal services and views of health professionals. PLoS One. 2014; 9(7): e103085, doi: 10.1371/journal.pone.0103085, indexed in Pubmed: 25058333.

13. Drews, K., Spaczyński, M., Brązert, J. : Rekomendacje Zarządu Głównego PTG w zakresie opieki przedporodowej w ciąży o prawidłowym przebiegu. Ginekol Dypl 8: 59-66. ; 2006.

14. Johnson K, Posner SF, Biermann J, et al. Recommendations to improve preconception health and health care - United States. Morb Mortal Wkly Rep (MMWR) 55: 1-23. ; 2006.

15. Brun-MicaleffE, Coffy A, Rey V, et al. Cervical cancer screening by cytology and human papillomavirus testing during pregnancy in French women with poor adhesion to regular cervical screening. J Med Virol. 2014; 86(3): 536-545, doi: 10.1002/jmv.23764, indexed in Pubmed: 24114972.

16. Wang Z, Yan $\mathrm{R}, \mathrm{He} \mathrm{H}$, et al. Difficulties in eliminating measles and controlling rubella and mumps: a cross-sectional study of a first measles and rubella vaccination and a second measles, mumps, and rubella vaccination. PLoS One. 2014; 9(2): e89361, doi: 10.1371/journal.pone.0089361, indexed in Pubmed: 24586717.

17. Pitkin RM. Folate and neural tube defects. Am J Clin Nutr. 2007; 85(1): 285S-288S, indexed in Pubmed: 17209211.

18. Mills JL. Malformations in infants of diabetic mothers. Teratology. 1982 25(3): 385-394, doi: 10.1002/tera.1420250316, indexed in Pubmed: 7051398.

19. Wender-Ozegowska E, Gutaj P, Szczepanek U, et al. Influence of pregnancy planning on obstetrical results in women with pregestational diabetes mellitus. Ginekol Pol. 2010; 81(10): 762-767, indexed in Pubmed: 21117305

20. Ripabelli G, Cimmino L, Grasso GM. Alcohol consumption, pregnancy and fetal alcohol syndrome: implications in public health and preventive strategies. Ann Ig. 2006; 18(5): 391-406, indexed in Pubmed: 17089955.

21. Grainger DA, Frazier LM, Rowland CA. Preconception care and treatment with assisted reproductive technologies. Matern Child Health J. 2006; 10(5 Suppl): S161-S164, doi: 10.1007/s10995-006-0094-y, indexed in Pubmed: 16802186.

22. Andiappan H, Nissapatorn V, Sawangjaroen N, et al. Toxoplasma infection in pregnant women: a current status in Songklanagarind hospital, southern Thailand. Parasit Vectors. 2014; 7: 239, doi: 10.1186/1756-33057-239, indexed in Pubmed: 24886651.

23. Sekhavat L, Davar R, Hosseinidezoki S. Relationship between maternal hemoglobin concentration and neonatal birth weight. Hematology. 2011; 16(6): 373-376, doi: 10.1179/102453311X13085644680186, indexed in Pubmed: 22183073.

24. Bakacak M, Avci F, Ercan O, et al. The effect of maternal hemoglobin concentration on fetal birth weight according to trimesters. J Matern Fetal Neonatal Med. 2014; 11: 1-5.

25. Romero R, Oyarzun E, Mazor M, et al. Meta-analysis of the relationship between asymptomatic bacteriuria and preterm delivery/low birth weight. Obstet Gynecol. 1989; 73(4): 576-582, indexed in Pubmed: 2927852.

26. Dias-Damé JL, Cesar JA. Disparities in prevalence of smoking and smoking cessation during pregnancy: a population-based study. Biomed Res Int. 2015; 2015: 345430, doi: 10.1155/2015/345430, indexed in Pubmed: 26075231.

27. Bolumar F, Rebagliato M, Hernandez-Aguado I, et al. Smoking and drinking habits before and during pregnancy in Spanish women. J Epidemiol Community Health. 1994; 48(1): 36-40, indexed in Pubmed: 8138766. 